\title{
Curiosidades na nomenclatura mineralógica: Porpezita do Brasil
}

\author{
Curiosities of the mineralogical nomenclature: Porpezite from Brazil
}

Hubert Mathias Peter Roeser ${ }^{1}$ e Kay Schürmann²

1 Depto. Eng. Ambiental, Escola de Minas, Univ. Fed. Ouro Preto, Campus Univ. s/n, 35.400-000 Ouro Preto, MG, Brasil. hubert-deamb@em.ufop.br 2 Senckenberg Forschungsinstitut und Naturmuseum, Senckenberganlage 25, D 60325 Frankfurt am Main, Alemanha.

\section{Manuscrito: \\ Recebido: 21/8/2013 \\ Corrigido: 12/9/2014 \\ Aceito: 11/4/2014}

Citation:Roeser H.M.P., Schürmann K. 2015. Curiosidades na nomenclatura mineralógica: Porpezita do Brasil. Terræ Didatica, 11(1):14-20. < http://www.ige. unicamp.br/terraedidatica/> .

Keywords: Gold, Palladium, Porpezite, Goias, Brazil

A nomenclatura mineralógica pode parecer à primeira vista um pouco confusa. Há vários aspectos que são usados na denominação de minerais: pessoas, acontecimentos, animais, lugares, propriedades físicas e químicas. Em tempos passados utilizavam-se principalmente as línguas latim e/ou grego. Em tempos atuais: inglês, alemão, francês, espanhol, português e até chinês. Entretanto, para a grande maioria dos cerca de 4.700 minerais atualmente conhecidos, é válido que, de acordo com a nomenclatura, eles possam ser classificados em quatro grupos:

1. Minerais podem ser nomeados por localidades, ou seja, segundo o lugar onde o mineral foi encontrado pela primeira vez (locus tipicus- localidade tipo):

Exemplos para este grupo são:

Andaluzita $\left(\mathrm{Al}_{2} \mathrm{SiO}_{5}\right)$, descrita pela primeira vez em Andaluzia, na Espanha.

Anglesita $\left(\mathrm{PbSO}_{4}\right)$, segundo a ilha de Anglesey em Gales, Inglaterra.

Antarticita $\left(\mathrm{CaCl}_{2} \cdot 6 \mathrm{H}_{2} \mathrm{O}\right)$, um mineral muito raro, que foi achado na década de 60 do século passado no lago Don Juan na Antártica, cuja salinidade extrema é de cerca de 44,2\% (Torii e Ossaka 1965).

Clausthalita (PbSe) é uma reminiscência da antiga cidade de mineração de Clausthal, Zellerfel, na montanha Harz, na Alemanha.

Elbaíta é uma turmalina de $\mathrm{Na}$ e Li, descrita pela primeira vez na Ilha de Elba, na Itália.

Exemplos de minerais brasileiros que se encontram neste grupo são:

Minasgeraisita $\left(\mathrm{CaBe}_{2} \mathrm{Y}_{2} \mathrm{Si}_{2} \mathrm{O}_{10}\right)$, que foi encontrada pela primeira vez no pegmatito Jaguaraçu (também conhecido como mina do José Miranda) no estado de Minas Gerais (IMA 2009).

Tripuhyita $\left(\mathrm{FeSbO}_{4}\right)$, descoberta no final do século 19 pelo mineralogista austríaco Eugenio Hussak no vale de Tripuí (naquela época escrito Tripuhy), próximo a Ouro Preto, MG. 
2. Minerais podem ser nomeados segundo pessoas. Às vezes conforme seu descobridor ou mesmo como uma homenagem a terceiros, assim, $\mathrm{FeO}(\mathrm{OH})$ recebeu o nome Goethita, segundo o poeta alemão Johann Wolfgang von Goethe [1749 - 1832], e Covellita (CuS) lembra o famoso mineralogista italiano Niccolo Covelli [1790 - 1829].

Nomes de minerais brasileiros imortalizados nesta categoria são, entre outros:

Gorceixita $\left(\mathrm{Al}_{3} \mathrm{H} \mathrm{Ba}\left[(\mathrm{OH})_{6} /\left(\mathrm{PO}_{4}\right)_{2}\right]\right)$ - é uma homenagem ao mineralogista francês Henri Gorceix (1842-1919), fundador da Escola de Minas de Ouro Preto, hoje integrada à Universidade Federal de Ouro Preto, UFOP, MG/Brasil.

Andradita $\left(\mathrm{Ca}_{3} \mathrm{Fe}_{2}\left[\mathrm{SiO}_{4}\right]_{3}\right)$ é o nome que lembra o cientista e estadista brasileiro José Bonifácio de Andrada e Silva (1763) e o fosfato barbosalita $\left(\mathrm{Fe}^{2+} \mathrm{Fe}^{3+}{ }_{2}\left(\mathrm{PO}_{4}\right)_{2}(\mathrm{OH})_{2}\right)$ comemora o professor de geologia da Escola de Minas em Ouro Preto -Aluízio Licínio de Miranda Barbosa (1916).

Outros exemplos de minerais brasileiros desta categoria são apresentados por Atencio (2000) e Gait (2000).

3. Minerais podem ser nomeados segundo as suas composições químicas, muitas vezes por nomes em latim.

São citados aqui em primeiro lugar os minerais nativos cujos símbolos químicos derivam-se diretamente do latim, como ouro (aurum - $\mathrm{Au}$ ), prata (argentum - $\mathrm{Ag}$ ), cobre (cuprum - $\mathrm{Cu}$ ), ferro (ferrum - Fe) etc. Como exemplos de minerais compostos por mais de um elemento temos o sulfeto de prata, denominado argentita $\left(\mathrm{Ag}_{2} \mathrm{~S}\right)$ (ainda considerando a associação entre prata/argentum, o leitor é lembrado do nome do Rio de la Plata, e da Argentina)

Estibnita $\left(\mathrm{Sb}_{2} \mathrm{~S}_{3}\right)$ é um sulfeto formado a partir de enxofre e antimônio, lembrando que antimônio em latim é antimonum, ou mesmo stibium.

Cuprita $\left(\mathrm{CuO}_{2}\right)$ vem do Cuprum (cobre), que também faz a raiz linguística para Chipre (depósitos de cobre).
Enquadram-se aqui muitos minerais brasileiros, como palladseita $\left(\mathrm{Pd}_{17} \mathrm{Se}_{15}\right)$, stibiopalladinita $\left(\mathrm{Pd}_{5} \mathrm{Sb}_{2}\right)$, arsenopalladinita $\left(\mathrm{Pd}_{8} \mathrm{As}_{3}\right)$ entre outros, todos encontrados pela primeira vez em concentrados de bateia em amostras do museu mineralógico da Escola de Minas em Ouro Preto, MG (Clarke et al. 1974, Roeser e Schürmann, 1990).

Outras duas espécies desta série de minerais $P d$, do museu mineralógico da Escola de Minas em Ouro Preto, são os minerais atheneita $\left((\mathrm{Pd}, \mathrm{Hg})_{3} \mathrm{As}\right)$, que homenageia a deusa grega Pallas Athena e a isomertieita $\left(\mathrm{Pd}(\mathrm{SbAs})_{2}\right)$ assim chamada por sua relação estrutural e composicional para a mertieita $\left(\mathrm{Pd}_{11}(\mathrm{Sb}, \mathrm{As})_{4}\right)$ denominada segundo o geólogo norte-americano John B. Mertie (1888-1980).

4. Minerais podem ser nomeados de acordo com suas propriedades físicas. Neste casos, os conceitos usados muitas vezes são derivações gregas.

Um belo exemplo é a cianita também chamada distênio. O primeiro nome deste silicato de alumínio $\left(\mathrm{Al}_{2} \mathrm{SiO}_{5}\right.$ - triclínico) é uma reminiscência do grego cianos (azul), lembrando a cor comum deste mineral, e seu segundo nome é também derivado do grego: distênio vem de distenos (duas forças), mencionando neste caso as duas principais direções de dureza do mineral.

O piroxênio enstatita $\left.(\mathrm{Mg}, \mathrm{Fe})_{2} \mathrm{SiO}_{6}\right)$, do grego enstates (oponente), foi denominado assim já em 1855 pelo mineralogista alemão Gustav Adolf Kenngott [1818-1897], porque ele constatou uma dificuldade em fundir o mineral com o teste de maçarico.

Estaurolita $\left((\mathrm{FeMg})_{2} \mathrm{Al}_{9} \mathrm{O}_{6}\left[\mathrm{SiO}_{4}\right]_{42}(\mathrm{OH}, \mathrm{O})_{2}\right.$ refere-se aos gêmeos cruciformes típicos deste mineral. Do grego stauros (cruz) e lithos (rocha, pedra).

Infelizmente encontram-se na literatura e, especialmente hoje na Internet, muitas interpretações errôneas. Assim, o mineral estroncianita $\left(\mathrm{SrCO}_{3}\right)$ não recebeu seu nome como frequentemente divulgado por causa do elemento estrôncio. Friedrich Gabriel Sulzer [1749-1830] denominou-o segundo o lugar onde estroncianita foi encontrada pela primeira vez, Scottish Loch Strontian. Posteriormente o elemento encontrado no mineral levou o seu nome, estrôncio.

Similar o caso da magnetita $\left(\mathrm{Fe}_{3} \mathrm{O}_{4}\right)$, e do magnetismo. A origem do nome da própria magnetita não é totalmente clara. Alguns autores a associam com a localidade de Magnesiana (Macedônia). Outros a atribuem ao um pastor grego chamado 
Magnes. Ele teria descoberto que o mineral atraiu os pregos de seus sapatos e a fivela do seu cinto. De qualquer maneira, este é mais um caso em que primeiro o mineral recebeu o seu nome e só então foi definido o fenômeno físico, neste caso o magnetismo, observado nele.

Cerca de 20 anos atrás já havia esforços internacionais para padronizar a nomenclatura mineralógica - reduzindo-a somente às propriedades físicas e químicas dos minerais. Assim uma comissão da Associação Internacional de Mineralogia (International Mineralogical Association - IMA), recomendou que não mais se nomeasse minerais segundo pessoas, mas apenas considerando suas composições químicas e características físicas (Nickel, 1995). Mas essas recomendações não se mantiveram.

Especialmente nomear um mineral segundo uma pessoa continua muito em uso. Ainda muitas vezes alunos honram assim seus professores, imortalizando-os na forma de nomes mineralógicos. Aqui somente dois exemplos de tempos recentes:

A arapovita $\left((\mathrm{U}, \mathrm{Th})(\mathrm{Ca}, \mathrm{Na})_{2}\left(\mathrm{~K}_{1-\mathrm{x}}[]_{\mathrm{x}}\right)\right.$ $\left.\mathrm{Si}_{8} \mathrm{O}_{20} \bullet \mathrm{H}_{2} \mathrm{O}\right)$ nomeada segundo Yu. A. Arapov [1907-1988], um geólogo russo, autor de muitos trabalhos geoquímicos na Ásia Central. E a lindbergita $\left(\left(\mathrm{Mn}\left(\mathrm{C}_{2} \mathrm{O}_{4}\right) \cdot 2\left(\mathrm{H}_{2} \mathrm{O}\right)\right)\right.$ que homenageia a geóloga Marie Louise Lindberg-Smith [1918 -] do USGS (Baumgärtl e Mücke, 2005).

Um exemplo bastante curioso neste sentido é o mineral armacolita $\left((\mathrm{Mg}, \mathrm{Fe}) \mathrm{Ti}_{2} \mathrm{O}_{5}\right)$ que foi trazido em 1969 da Lua pelos primeiros astronautas americanos e consequentemente levou o nome deles: Neil Armstrong, Edwin E. Aldrin Jr. e Michael Collins.

Tem-se muitas vezes a impressão de que hoje em dia não há mais limites de ideias para nomeações de minerais. Como no caso dos selos postais comemorativos, aparecem minerais nomeados segundo todas as possibilidades. A Grande Muralha da China reflete-se na changchengita (IrBiS). Mutinaita $\left(\mathrm{Na}_{3} \mathrm{Ca}_{4} \mathrm{Al}_{11} \mathrm{Si}_{85} \mathrm{O}_{192} \bullet 60 \mathrm{H}_{2} \mathrm{O}\right)$, embora encontrado na Antártida, menciona o centro italiano de pesquisas sobre zeólitas, Mutina. Também a descoberta secular dos raios - X por Konrad Wilhelm Röntgen [1845 - 1923], foi comemorada em todo o mundo, não só com selos especiais, mas também com o novo mineral caoxita ( $\mathrm{Ca}$ $\left.\left[\mathrm{C}_{2} \mathrm{O}_{4}\right] \cdot 3 \mathrm{H}_{2} \mathrm{O}\right)$. O conceito inglês caoixte menciona Centennial Anniversary of $X$ rays (aniversário centenário dos raios-X). Pelo menos neste caso a coaxita apresenta aspectos químicos, pois pode ser interpretado também quimicamente como calcium

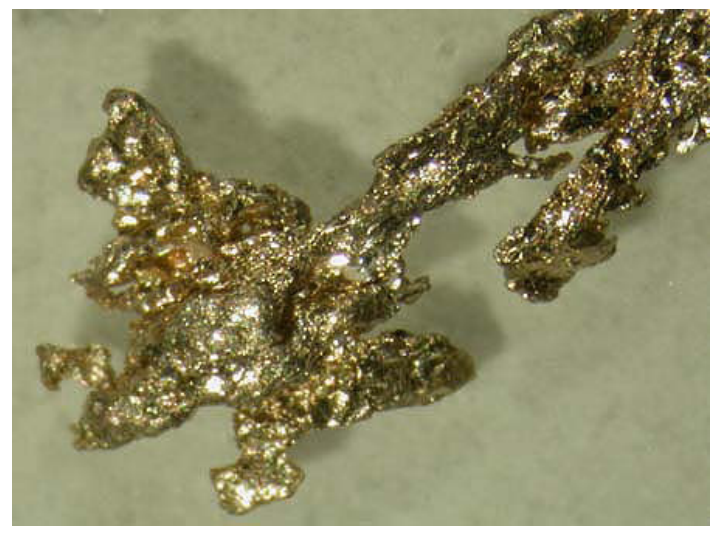

Figura 1. Porpezita (AuPd) de Itabira, Minas Gerais, Brasil Cortesia de Diederik Visser (http://www.dvminerals.com) A largura da imagem corresponde aproximadamente a $6 \mathrm{~cm}$

oxalate (oxalato de cálcio) (IMA 2009).

Existem até minerais que portam nomes de animais como aoursinita $\left(\mathrm{C}(\mathrm{Co}, \mathrm{Mg})\left(\mathrm{H}_{3} \mathrm{O}\right)_{2}[\right.$ $\left.\left.\left(\mathrm{UO}_{2}\right) \mathrm{SiO}_{4}\right]_{2} \bullet 3\left(\mathrm{H}_{2} \mathrm{O}\right)\right)$ do francês 1'oursin (ouriço do mar), uma referência ao seu hábito, e a corvusita $\left(\mathrm{NaCa}\left(\mathrm{V}^{5+} \mathrm{V}^{4+} \mathrm{Fe}^{2+}\right)_{8} \mathrm{O}_{20} \cdot 4 \mathrm{H}_{2} \mathrm{O}\right)$ do latim corvus (corvo), por causa da cor semelhante entre o mineral e o pássaro, mas mesmo nestes casos deve ser mantida certa cautela com interpretações: escorpionita $\left(\mathrm{Ca}_{3} \mathrm{Zn}_{2}\left(\mathrm{PO}_{4}\right)_{2} \mathrm{CO}_{3}\right.$ $\left.(\mathrm{OH}) 2 \cdot \mathrm{H}_{2} \mathrm{O}\right)$, por exemplo, leva o nome de uma mina de Zn na Namíbia (Skorpionzincdeposit, Namibia) (IMA 2009).

Um nome um tanto incomum é apresentado a seguir. Durante as nossas investigações sobre o ouro paladiado do Quadrilátero Ferrífero, em Minas Gerais, Brasil, encontramos várias vezes na literatura científica o nome Porpezita. Este termo é aplicado ao mineral de fórmula química AuPd (fig. 1). Como mostra a figura, este ouro paladiado é mais branco do que o "ouro normal" (contendo principalmente $\mathrm{Cu}$ ), o que já salienta Hussak em sua dissertação de 1904. Couto (1799) até confundiu este AuPd por causa de sua cor clara com platina. Vários grãos deste mineral foram analisados desde o início da década de 90 (resumo em Roeser e Schürmann 2008).

$\mathrm{Na}$ virada dos séculos 19 - 20, especialmente nos primeiros anos do século 20 , porpezita emerge repetidamente nos livros de mineralogia, em particular na Europa e especialmente na literatura francesa, por exemplo. (Charpentier 1900).

No livro Précis de Minéralogie do ano 1908 do Francês De Lapperent, o autor coloca porpezita (Porpézite,or allié de palladium) na lista de espèces et variétés. 
Pisani (1883) indica dados químicos e mencionou que la Porpézite ou Or palladié (de Porpez, au Brésil) contient 4 p. 100 d'argent et 10 p. 100 de palladium. Semelhantemente escreve Buttgenbach (1923): la Porpézite contient 4\% d'argent et 10\% de palladium.

A composição química da porpezita foi indicada por vários autores em valores similares, que leva à conclusão de que um copia do outro. Naumann (1881) escreveu na décima primeira edição do seu livro Elemente der Mineralogie (Elementos da Mineralogia): Porpezita (Palladiumgold) é uma variedade de ouro que foi denominado segundo sua área de ocorrência, a Capitania Porpez no Brasil, e que contem além de 4 p.Ct prata quase 10 pCt Paládio (pCt significa aqui \%).

Provavelmente todas essas passagens se referem à primeira análise química do ouro-paládiado (porpezita), que já foi realizada em 1835 pelo químico sueco Jöns Jacob Berzelius, mais conhecido como Jacob Berzelius [1779-1848].

Outras citações podem ser encontradas nos artigos e obras de DeLapperent (1908 b), Niggli (1926), Vaz (1928, 1953), Picone Flahaut (1957) entre outros.

É interessante constatar que alguns autores logo duvidaram da existência da porpezita como um mineral próprio, pois em muitos manuais conhecidos de mineralogia da época, como Miers (1929), o nome é omitido. A Associação Mineralógica Internacional (IMA) nunca reconheceu o nome porpezita. Em seu banco de dados (www. webmineral.com), assim como na Mineralogia ATHENA, ou no Danade 1997, que reserva para o ouro apenas uma página e meia, a porpezita não é mais listada. Também falta o nome porpezita em dois trabalhos mais recentesque tratam de minerais brasileiros (Atencio 2000, Gait 2000).

Deixando essas discussões de lado, o interesse aqui é apenas relacionado ao processo de nomeação da porpezita, o que possui certo aspecto humorístico devido ao fato de que seu nome não se enquadra em nenhuma das classificações descritas anteriormente: localidades, pessoas, composições químicas, etc. Não há propriedade física ou química, com qual poderia ser ligada ao nome desse mineral. Falta também um nome de celebridade relevante igual Porper, Porpez etc.. Além disso, apesar das localidades listadas na literatura citada acima, no Brasil nunca existiu um lugar que está ligado ao nome, especialmente uma Capitania Porpez.

Em conversas com os colegas brasileiros, especialmente os formados há mais tempo, nas déca- das de 70 e 80 do século passado, veio à tona uma história que a primeira vista parece uma anedota, mas que é confirmada em pelo menos duas breves referências bibliográficas, que dizem que o termo porpezita é um erro não intencional, ou seja, uma desfiguração de outro nome. Em relação ao tal nome original, posteriormente desfigurado, a literatura geológica cita dois lugares:

Leonardos (1973) correlaciona no seu livro "Geociências no Brasil - Uma Contribuição Germânica o nome porpezita com a mina Pompeu perto de Sabará, em Minas Gerais. Provavelmente ele se refere a uma comunicação oral de Derby para Dana, que já está listada na $6{ }^{\text {a }}$ edição do Sistema de Dana de Mineralogia em 1892 : Palladium-gold (Porpezite Fröbel). A variety from Porpez, Brasil, containing 10 p.c. of palladium, besides some silver, color pale; also from Jacutinga and Condonga with 5 to 6 p.c. Pd. Porpez however, is probably a corruption of Pompeo, an old mining settlement near Sabará, in which vicinity palladium-gold occurs rather abundantly (Derby, Priv. Contr.).

Ou seja: ouro-paladiado (porpezita em Fröbel) é uma variedade de Porpez, Brasil, contendo 10 pc de paládio, além de algumas partes de prata, cor pálida, também conhecida de Jacutinga e Condonga com 5-6 pc Pd. Porpez, no entanto, é provavelmente uma corruptela de Pompeo, uma antiga mineração, perto de Sabará, onde ouro-paladiado ocorre abundantemente. (Derby, Priv Contr.). Tal explicação encontra-se até hoje em alguns sites da Internet, por exemplo em: http://www.mindat.org/ min-10923.html: porpezite: name: NamedbyFrobel (Dana6, p 53, (1892)) after a Brazilian locality now lost. Dana7, p 91, (1944) references ["Porpez" (Pompeo ?), Taguaril and other localities in Minas Gerais and Goyaz, Brazil].

Assim, Dana assume uma cópia defeituosa (corruption) da palavra Pompeo. Hussak (1904) e Ramdohr (1948) concordam com tal desfiguração, mas eles apresentam para a localidade uma segunda explicação que parece ser mais plausível.

A literatura mais antiga menciona que o ouro contendo paládio, conhecido também como ouro podre e às vezes por causa de sua cor brilhante confundido com platina, já surgiu no Brasil no final do século XVII (Calógeras 1904). O elemento paládio foi, no entanto, só descoberto como elemento próprio em 1803, em pepitas de AuPd em conjunto com platina. Muitos autores se referem ás primeiras descobertas na Colômbia, mas conforme Hussak (1904) e Leonardos (1973), as primeiras pepitas de platina contendo paládio vieram do 
Brasil. Hussak lembra neste contexto o mineralogista austríaco Pohl, que levou amostras de ouro da província brasileira de Goiás para Viena: O ouro analisado vem das coleções do geólogo vienense Pohl, que viajou pelo estado brasileiro de Goiás (anteriormente os estados eram designados como capitania, mais tarde como província e agora como estados). Pohl não menciona em seu livro "Contribuições para a orografia do Brasil" a ocorrência deste ouro. Mas, como se sabe, suas coleções são guardadas no Real Museu de História Natural em Viena desde 1837. E nelas foi encontrado sob o $n^{\circ} 1099$ e 1100, museu brasileiro, um ouro designado como PdAu, originado de Arrayas, capitania de Goyaz.

Após a descoberta do AuPd e, assim do Pd, provavelmente se realizou agora uma busca intensa de amostras brasileiras contendo Pd, como mostram referências bibliográficas, por exemplo, em Lampadius e Johnson (1837). Em tais casos é usual que as amostras também sejam intercambiadas entre pessoas e instituições. Neste caso, não é possível identificar claramente o que aconteceu. Pode ter ocorrido, por exemplo, problemas durante tal intercâmbio (troca de etiquetas!?) ou as etiquetas foram perdidas. Hussak (1904) observou em duas amostras o nome Goyaz, mas talvez os outros rótulos não estivessem tão claramente descritos. De qualquer maneira aconteceu aqui uma distorção associada a esse nome Goyaz, como também imaginou Ramdohr em 1948: O ouro também raramente contém Pd (ouro paládio ou Porpezita de Goyaz, erradamente de Porpez no Brasil). Essa desfiguração de Goyaz para Porpez está associada muitas vezes ao nome do mineralogista Friedrich Wilhelm August Fröbel [1782 -1852].

Existem três possibilidades de como o Goyaz se transformou em Porpez (vide figura 2) em nossa opinião:

1- Em primeiro lugar, deve-se ter em mente as modestas condições técnicas daquele tempo, em que se escrevia com pena de ganso em um papel de qualidade não muito boa, com uma tinta que com certeza não possuía as qualidades das tintas atuais. A alta umidade dos trópicos certamente contribuiu para que as letras ficassem borradas e os rótulos fossem dificilmente identificáveis.

2- Outra possibilidade é que as peças dos pesquisadores foram descritas por caboclos, pois era comum naquele tempo que os exploradores trouxessem seus serventes com eles, ou mesmo contratassem assistentes locais. Estes eram geralmente pessoas humildes, várias vezes com pouca instrução. Eles realizavam todos os tipos de trabalhos. Basta notar que até hoje existem muitos casos de pessoas simples do interior que ainda tem dificuldades de escrever. Assim, imaginase, que aqui também abre-se uma possível fonte para a ilegibilidade eventual de alguns rótulos e uma má interpretação do escrito consequente.

3- Uma terceira alternativa, que também não pode ser completamente descartada, seria a influência da cachaça (aguardente de cana) sobre a caligrafia no rótulo da amostra (Fig. 2). Também em Goiás cultivou-se na época a cana de açúcar da qual foi e é destilada a bebida nacional brasileira. Será que a forma leve de um delirium tremens temporário (tremens, do latim - tremendo!) tenha influenciado a formação de um nome mineralógico?

Deixamos para o leitor interessado de nestas linhas aplicarem testes adequados e, até mesmo elaborar um diagrama de correlação entre teor alcoólico no sangue versus caligrafia do nome de Goyaz.

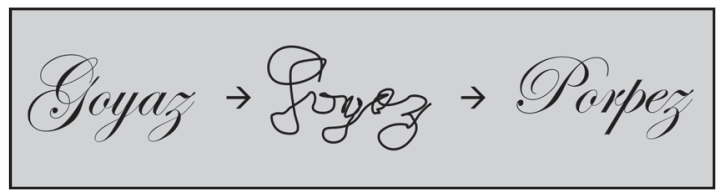

Figura 2. Possível influência da cachaça (aguardente de cana) sobre a caligrafia

Como pode se ver a história da "porpezita" possui certo aspecto humorístico.

Finalmente, não podemos deixar de mencionar o adágio de: Quem é declarado morto, vive mais. Pois parece que a porpezita ainda não morreu completamente. Especialmente na Internet, ela festeja uma ressureição como mostram milhares de websites. Basta ver os resultados de uma pesquisa no Google neste sentido:

Português: porpezita - aproximadamente 566 resultados em 0,33 segundos.

Inglês: porpezite - aproximadamente 56.600 resultados em 0,31 segundos.

Alemão: Porpezit - aproximadamente 2.840 resultados em 0,35 segundos; 
Frances: porpezite - aproximadamente 5.290 resultados em 041 segundos, etc. etc.

Aqui só três destes exemplos:

No site do Museu Heinz Ebert da UNESP: http://www.rc.unesp.br/museudpm/banco/ elementos\%20nativos/paladio.html encontra-se sob o mineral Paládio: [...] Associação - Forma um selenieto e aparece nos minérios sulfetados de níquel; também nos minerais stíbio-paladinita, braggita e porpezita.

No léxico de joias do professor Leopold Roessler (http://www.beyars.com), (não confundir com o nome do primeiro autor!), encontra-se: Porpezit : Natürliches palladiumhältiges Gold. (Porpezita ouro paládiado natural).

No site espanhol http://mundo-mineral.blogspot.com/search/label/Porpezita

Variedades: Electrum: con un 25 a 28\% de plata.

Porpezita: Au aleado con paladio (Goya, Brasil) sic!

Encontra-se neste último site até uma nova capitania brasileira, desta vez não Porpez mais Goya! Esse é mais um exemplo de que a internet pode nos oferecer um serviço inestimável, se nós soubermos lidar com ela.

\section{Agradecimentos}

Os autores agradecem a Patricia Angelika Roeser (Universidade de Bonn, RFA) e o Erik Sartori Jeunon Gontijo (Universidade Federal de Ouro Preto, Brasil) pela leitura crítica do artigo.

\section{Referencias bibliográficas:}

Atencio D. 2000. Minerals for which Brazil is the type locality. Rocks \& Minerals, 75(1):44-46.

Baumgärtl U., Mücke A. 2005. Neue von der IMA (International Mineralogical Association) anerkannte Mineralien. Heidelberg: Eine Kurzfassung (30) Aufschluss, 56, NM 213-NM 216.

Buttgenbach, H. 1923. Les Mineraux et les Roches. 3o Éditon, 629 Seiten + Compléments 107 Seiten, Dunand Éd., Paris, Liége: H. Vaillant-Carmann Imprimeur.

Calógeras J.P. 1904. As minas do Brasil e sua legislação. Rio de Janeiro: Imprensa Nacional, v. I, 477p.

Charpentier H. 1900. Géologie et Minéralogie Appliquées. Les Minéraux utiles \& leurs Gisements Vve Ch. Paris: Dunod Éd. 643p.

Clark A.M., Criddle A.J., Fejer E.E. 1974. Palladium arsenide, antimonides from Itabira, Minas Gerais, Brazil. Miner. Mag., 39:528-543.

Couto J.V. 1799. Memoria sobre a Capitania de Mi- nas Geraes, seu territorio, clima e produções metalicas; sobre a necessidade de se restabelecer e animar a mineração decadente do Brasil; sobre o commercio e exportação de metaes e interesses regios. Com um appendice sobre os diamantes e nitro natural. Tudo por ordem de Sua Majestade. Anno de 1799, 194 p., originalmente manuscrito inédito. [Este manuscrito foi impresso em 1848 na Revista Trimestral do Instituto Histórico Etnográfico Geográfico Brasileiro. t XI, 289-335, Rio de Janeiro. Existe tradução em Inglês de 1904, publicada nos Anais da Biblioteca de Ciência do Museu Victoria e Alberto (Science Library of the Victoria and Albert Museum London), setembro, 29, 1904].

De Lapparent A. 1908. Cours de Minéralogie. 4a édition, Masson et Cie. Éditeurs, 413 páginas, Paris.

De Lapparent, A. 1908b. Précis de Minéralogie. Paris: Masson et Cie. Éditeurs, 756p.

Gait R.I. 2000. Who's Who in Mineral Names: People after Whom Type Minerals from Brazil Have Been Named. Rocks \& Minerals, 75(1):47-49.

Hussak E. 1904. Über das Vorkommen von Palladium und Platin in Brasilien. Sitzungsberichte Kais. Akademie der Wissenschaften, Math. Naturw. Klasse, Bd. CXIII, Abt. I, 88 páginas, Wien.

Lampadius W.A., Johnson D.N. 1837. Über brasilianisches Palladgold und dessen Ausbringung und Scheidung. (Mittheilung des Herrn D.N. Johnson nebst Bemerkungen von W.A. Lampadius). Leipzig: J. für praktiscche Chemie, 2. Bd., 309-315.

Leonardos O.H. 1973. Geociências no Brasil. A Contribuição Germânica. Rio de Janeiro Forum Sulina Ed. 345p.

Miers H. 1929. Mineralogy. An introduction to the scientific study of minerals. London: MacMillan Co. Ltd. 658 p.

Nickel E.H. 1995. The definition of a mineral. Montreal: Can. Miner., 33:689-690.

Nickel E.H., Mandarino J.A. 1987. Procedures involving the IMA Commission on New Minerals and Mineral Names and guidelines on mineral nomenclature. Am. Miner., 72(9-10):1031-1042.

Niggli P. 1926. Lehrbuch der Mineralogie II. Spezielle Mineralogie. 2 Auflage. Berlin: Verlag Gebrüder Bornträger. 697p.

Naumann C.F. 1881. Elemente der Mineralogie. 11 Auflage. Leipzig: Verlag Von Wilhelm Engelmann. 735 p.

Picon M., Flahaut J. 1957. Éléments de Minéralogie et de Cristalographie. Paris: Soc. D’Éditeurs D’Énseignement. 293p.

Pisani M. F. 1883. Traité Élémentaire de Minéralogie. Paris: G. Masson Éd. 421p.

Ramdohr P. 1948. Klockmann's Lehrbuch der Mineralogie.13 Auflage. Stuttgart: Ferdinand Enke 
Verlag. 674p.

Roeser H., Schürmann K. 1990. Atheneit aus dem Eisernen Viereck, Zentralbrasilien. 12 Geow. DFG Kolloqium, Zusammenfassungen, München.

Roeser H., Schürmann K. 2008. Die Geschichte des schwarzen Goldes (Ouro Preto) in Brasilien. Heidelberg: Aufschluss 59:273-294.

Torii T, Ossaka J. 1965. Antarcticite: A New Mineral, Calcium Chloride Hexahydrate, Discovered in Antarctica. Science. 149:975-97.

Vaz TH. 1928. Determinação de Mineraes. Ouro Preto: Ed. Livr. Mineira. 175p.

Vaz TH. 1953. Determinação de Minerais. Belo Horizonte: Estab. Gráf. Santa Maria. 200p.
Sítios WEB utilizados (em ordem alfabética): http://www.beyars.com/lexikon/lexikon_p_2.html http://www.dvminerals.com

http://susi.e-technik.uni-ulm.de:8080/Meyers2/seite/ werk/meyers/band/13/ seite/0237/

http://www.mineralatlas.com http://www.mineralienatlas.de/lexikon/index.php/ Brasilien/Minas\%20Gerais/Itabira http://www.webmineral.com (banco da IMA)

RESUMO: Na virada do século 19 para o século 20, especialmente nos primeiros anos do século 20, surgiu o nome Porpezita, imerso em livros de mineralogia, principalmente na Europa e em especial na literatura francesa. 0 termo foi atribuído à liga de ouro-paládio (Au, Pd). Amostras de Porpezita chegaram a Viena, em meados do século 19, provavelmente pelo mineralogista austríaco Pohl. Dizia-se que elas teriam chegado da província Porpez no Brasil. No entanto, essa capitania não existe e jamais existiu. 0 mineral recebeu seu nome provavelmente de uma desfiguração do nome do estado brasileiro de Goiás. Este artigo discute três possíveis razões para tal distorção, ou seja, de como Goiás virou Porpez e como Porpez deu seu nome ao mineral Porpezita. A IMA (International Mineralogical Association) não reconhece o nome; no entanto, ele não pode ser mais banido da literatura, particularmente da InterNet.

PALAVRAS CHAVE: Ouro, Paládio, Porpezita, Goiás, Brasil

ZUSAMMENFASSUNG : An der Wende des 19. zum 20. Jahrhundert, vor allem in den frühen Jahren des 20. Jahrhunderts, taucht in Mineralogie Büchern insbesondere in Europa und vor allem in französischer Literatur, immer wieder der Name Porpezit auf. Damit wurde die Gold - Palladiumlegierung Au,Pd, benannt. Proben von Porpezit gelangten Mitte des 19. Jahrhunderts wohl über den österreichischen Mineralogen Pohl nach Wien. Sie sollten aus der Capitania (Provinz) Porpez in Brasilien stammen. Allerdings gibt es eine solche Capitanie nicht und hat sie auch nie gegeben. Das Mineral verdankt seinen Namen sehr wahrscheinlich einer Entstellung des Namens des brasilianischen Bundesstaates Goias. Drei mögliche Gründe für eine solche Entstellung, wie aus Goias Porpez wurde, und wie Porpez dem Mineral Porpezit seinen Namen gab, werden hier diskutiert. Die IMA (International Mineralogical Association) erkennt diesen Namen nicht an. Dennoch ist er aus der Literatur, vor allem dem INTERNET nicht mehr zu verbannen.

SCHLÜSSELWORTE: Gold, Palladium, Porpezit, Goias, Brasilien 